Apidologie, 1971, 2 (2), 157-167.

\title{
MIKROSKOPISCHE BESTANDTEILE BRASILIANISCHER HONIGTAUHONIGE
}

Les constituants microscopiques des miels de miellat brésiliens

Ortrud Monika BARTH (')

Instituto Oswaldo Cruz, Rio de Janeiro (Brasilien)

SUMMARY

THE MICROSCOPIC COMPONENTS OF BRAZILIAN HONEYDEW HONEYS

The author describes the first microscopic examinations of brazilian honeydew honeys, which show a small amount of algae cells and sooty mould spores, but a large quantity of granulous aggregates and other plant fragments.

\section{ZUSAMMENFASSUNG}

Erste mikroskopische Beobachtungen an brasilianischen Honigtauhonigen lassen einen geringen Gehalt an Algenzellen und Russtausporen erkennen, jedoch reichhaltig granuläre Masse und sonstige Pflanzenfragmente.

Das Vorkommen von Honigtau in brasilianischen Bienenhonigen ist keine Seltenheit, dagegen dürften kaum Honige reiner Honigtautracht, so wie wir sie gut aus den mitteleuropäischen Nadelwaldbeständen kennen, anzutreffen sein. Es fehlt diesbezüglich jedoch für Brasilien jegliche Beobachtung, so dass im Rahmen der vorliegenden Arbeit nur ein kleiner Ausschnitt einer weitgreifenden Aufgabe behandelt werden kann.

(I) Mit Unterstützung des Conselho Nacional de Pesquisas. 
Vereinzelte, auf brasilianischen Honigtau hinweisende Angaben wurden von Barth (1970) zusammengestellt. Mikroskopische Beobachtungen an Honigtausedimenten liegen nicht vor. Der Reichtum an pflanzensaugenden Homopteren (Lima, 1936) ist unvorstellbar gross, jedoch ist ihre biologische Bedeutung grösstenteils unbekannt, so dass von hier aus gesehen keine Lösungsmöglichkeiten vorliegen. Russtaubefall von Pflanzenkulturen (z. B. an Citruspflanzen : Gonçalves, 1940) ist nicht selten, doch inwiefern dies zu einer Honigtautracht führt, ist nicht bekannt.

Mikroskopische Untersuchungen an ungefähr 90 Bienenhonigproben liessen bei etwa der Hälfte der Proben einen Nachweis von Honigtauelementen zu. An erster Stelle stehen Russtausporen, granuläre Masse, Russteilchen und Windblütlerpollen, an zweiter Algenzellen und sonstige Teile pflanzlicher Oberflächen.

Unter den Russtausporen trifft man am häufigsten solche des Coniothecium-Typs an, die seltener vereinzelt, meist in dichten, dunkelbraunen, formlosen Sporenballen auftreten (fig. 1, 2 und 3). Keimende Sporen und wachsende Hyphen sind nicht selten (fig. 4). Atichia-Sporenballen (fig. 5) kamen in einem Eucalyptus-Honigtauhonig und in einem Mischhonig vor. Weitere Sporentypen konnten nicht bestimmt werden (fig. 6). Eine Honigprobe unbekannter Herkunft aus Bahia enthielt ausser dem einen oder anderen Pollenkorn eine Vielzahl vom Uredosporen (fig. 7).

Häufig findet man in brasilianischen Mischhonigen eine granuläre Masse in lockerer, gleichmässiger Verteilung oder als kompakte, verklumpte Masse, in welcher Pollenkörner und Pilzsporen eingebettet liegen (fig. 8 und 9).

Selten dagegen treten Algenzellen auf. Meistens handelt es sich um grünliche Zellverbände der Protococcalen (fig. 10), weitaus seltener um Blaualgen (fig. 11) und Diatomeen. Pflanzendetrite, Zellteile, Zellwände, Steinzellen, Drüsenzellen und Pflanzenhaare (fig. 12, 13 und 14) sind fast stets vorhanden; in manchen verunreinigten Proben kamen auch Holzbestandteile vor.

Russ, in Form von kleineren oder grösseren Schollen, oder auch Russnadeln (von Grasbränden) treten sehr häufig in Honigtauhonigen auf (fig. 15 und 16), desgleichen auch Stärkekörner, vereinzelt oder in dichten Paketen gelagert (fig. 17). Weitere Bestandteile der Honigtauhonige sind Windblütlerpollen, an erster Stelle stehen Mais (wird meistens gehöselt; fig. 18), andere Gramineen des Paspalum-Typs und Cyperaceen (fig. 19), weiterhin Casuarina-, Cecropia- und Piperaceen-Pollen, sowie solcher vom Chenopodium-Typ (z. B. Amaranthus).

Eine Anzahl bekannter und unbekannter Bestandteile des Honigtaus, welche jedoch nicht als Indikatoren gelten, wurden in fig. 20-22 zusammengestellt.

Zusammenfassend ergeben diese ersten Beobachtungen an brasilianischen 
Honigtauhonigen, im Vergleich mit solchen ausländischer Herkunft (Maurizio, 1959), folgende Feststellungen :

1. Brasilianische Honigtauhonige zeichnen sich durch einen geringen Gehalt an Russtausporen (Coniothecium- und Atichia-Typen) aus.

2. Algenzellen sind sehr selten.

3. Pflanzen- und Zellfragmente treten häufig auf.

4. Granuläre Masse in lockerer oder kompakter Verteilung ist oft vorhanden.

5. Russteilchen verschiedenster Formen und Grössen sind häufig.

6. Pilzsporen und Hyphen aus Luftsedimenten sind nicht selten.

7. Windblütlerpollen ist stets vorhanden.

In Bezug auf pollenarme Honige bleiben folgende Fragen noch zu beantworten :

a) Wie können Honige von Blütennektarspendern, deren Pollen nicht gleichzeitig eingesammelt wird (Calliandra-Arten, Asclepiadaceen), bestimmt werden?

b) Gibt es Indikatoren für Honige aus extrafloralen Nektarien ?

c) Wie könnte Honig, der aus Pflanzenläsionen hervorquellenden Phloemsaft enthält, charakterisiert werden? (Beobachtungen zeigten, daß Meliponiden - stachellose Wildbienen - stark im Wachstum begriffene Bäume zwar nicht zu totalem Absterben bringen, aber doch bis zum Kahlwerden schädigen können, indem sie die Rinde junger $Z$ weige und frischer Triebe ringförmig abnagen.)

Eingegangen im September 1970.

Reçu pour publication en septembre 1970.

\section{RÉSUMÉ}

Les Homoptères parasites de plantes sont nombreux au Brésil. On sait cependant peu de chose sur leur importance biologique. De même, jusqu'ici, aucune recherche sur les miels de miellat brésiliens n'a été publiée. Les premières observations effectuées par l'auteur ont donné les résultats suivants :

1) Les miels de miellat brésiliens se distinguent par une faible teneur en spores de fumagines. On a trouvé surtout des spores du type Coniothecium (fig. I à 4), du type Atichia (fig. 5) ainsi que d'autres formes en partie indéterminables (fig. 6, 7, 20 à 22).

2) Les cellules d'algues sont très rares (fig. 10 et 11 ).

3) Les fragments de plantes et de cellules apparaissent souvent (fig. 12 à 14).

4) Une masse granuleuse plus ou moins compacte est souvent présente (fig. 8 et 9).

5) Des particules de suie de différentes formes et tailles ainsi que des grains d'amidon sont fréquents (fig. 15 à 17).

6) Les spores de champignons et les hyphes provenant de l'atmosphère ne sont pas rares. 
7) Les pollens de plantes anémophiles sont toujours présents (fig. 18 et 19).

En rapport avec les miels pauvres en pollen, les questions suivantes restent encore sans réponse : Comment peut-on identifier les miels de plantes fournissant du nectar mais dont le pollen n'est pas récolté en même temps que celui-ci (Espèces du genre Calliandra et Asclepiadacées)? - Existe-t-il des indicateurs pour les miels provenant de nectaires extra-floraux ? - Comment peut-on caractériser les miels contenant de la sève s'écoulant des lésions des plantes?

\section{LITERATUR}

BARTH, O. M., 1970 : Análise microscópica de algumas amostras de mel. 5. Melato (c honeydew ») em mel de abelhass. Rev. Brasil. Biol., 30, (4), 601-608.

Lima, A. da C., 1936 : Terceiro Catálogo dos Insetos que vivem nas Plantas do Brasil. - Escola Nacional de Agronomia. Diretoria de Estatística da Produção. Seção Publicidade. $460 \mathrm{~S}$.

Gonçalves, C. R., 1940 : Observações sôbre Pseudococcus comstocki (Kuw., 1902) atacando Citrus na Baixada Fluminense. - Rodriguésia, 13, S. 179-198.

Maurizio, A., 1959 : Zur Frage der Mikroskopie von Honigtau-Honig. - Ann. Abeille, 2, 145 158. 



\section{TAFEL 1}

Vergrösserungen, wenn nicht anders angegeben, $500 \times$

Авв. 1. - Sporenballen aus einem Eucalyptus-Honigtauhonig (Rio de Janeiro).

Aвв. 2. - Sporenballen aus einem schlecht riechenden und schmeckenden Borreria verticillata (Bv)-Honig (Ceará).

Aвв. 3. - Pilzsporen, Hyphen und Pollenkorn aus einem verunreinigten Honig (Ceará).

Авв. 4. - Gekeimte Pilzsporen aus einem verunreinigten Honig (Ceará).

Aвв. 5. - Sporenballen des Atichia-Typs und Vernonia (V)-Pollenkorn aus einem Eucalyptus-Honigtauhonig (Rio de Janeiro).

Авв. 6. - Helminthosporium (?) und andere unbekannte Pilzsporen. (700 x ; $P=1000 \mathrm{x})$.

Авв. 7. - Portulaca-Pollenkorn und Uredospore aus einem sehr pollenarmen Honig (Bahia).

Авв. 8. - Honigtauhonig mit geballter granulärer Masse (Rio de Janeiro) $(100 \mathrm{x})$.

\section{PLANCHE 1}

Sauf indication contraire, grossissement uniforme $\times 500$

Fig. 1. - Paquet de spores dans un miel de miellat d'Eucalyptus (Rio de Janeiro).

Fig. 2. - Paquet de spores dans un miel de Borreria verticillata (Bv), (Ceará). Ce miel est d'odeur et de goût désagréables.

FIG. 3. - Spores de champignons, hyphes et grains de pollen dans un miel souillé (Ceará).

Fig. 4. - Spores de champignons en germination dans un miel souillé (Ceará).

Fig. 5. - Paquets de spores du type Atichia et grain de pollen de Vernonia ( $V$ ) dans un miel de miellat d'Eucalyptus (Rio de Janeiro).

Fig. 6. - Helminthosporium (?) et autre spores de champignons inconnues $(700 \mathrm{x} ; P=1000 \mathrm{x})$.

Fig. 7. - Grain de pollen de Portulaca et Uredospore dans un miel très pauvre en pollen (Bahia).

FIg. 8. - Miel de miellat avec masse granuleuse par paquets (Rio de Janeiro) $(100 \mathrm{x})$. 

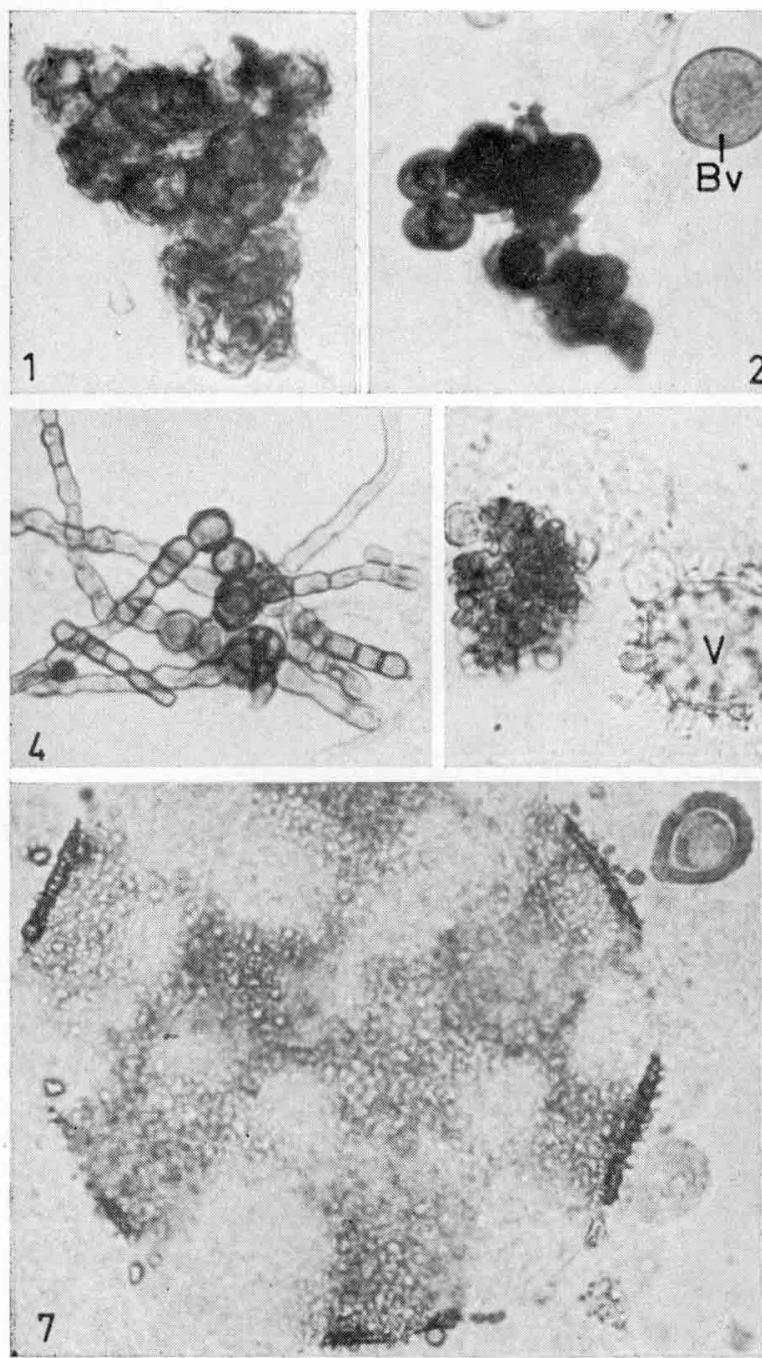

5

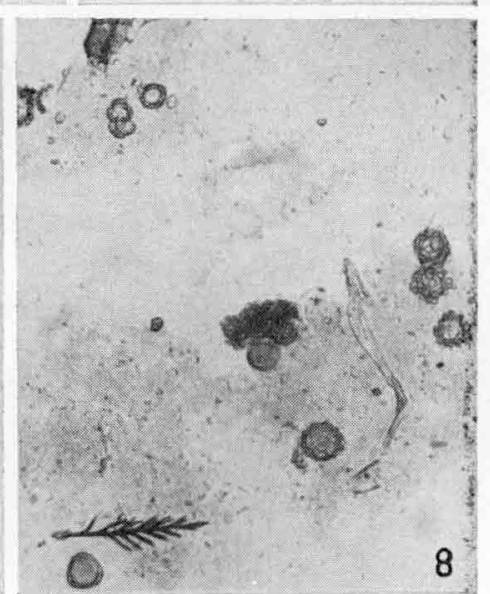

\section{Ortrud Monika Barth}


Aвв. 9. - Zuckerrohrhonig : granuläre Masse und Russpartikel (Minas Gerais). Aвв. 10. - Algenzellen (Honig aus Bahia).

Aвв. 11. - Blaualgenkolonie und Pilzsporen aus einem stärkereichen Honig (Paraná).

Aв8. 12. - Pflanzenfragment (Honig aus Rio de Janeiro).

Aвв. 13. - Pflanzenhaar aus einem verunreinigten Honig mit Pollenüberschuss (Bahia) (100 x).

\section{PLANCHE II}

Fig. 9. - Miel de canne à sucre : masse granuleuse et particules de suie (Minas Gerais).

Fig. 10. - Cellules d'algues (Miel provenant de Bahia).

Fig. 11. - Colonie d'algues bleues et spores de champignons dans un miel riche en amidon (Paraná).

Fig. 12. - Fragment vêgétal (Miel de Rio de Janeiro).

Fig. 13. - Poil végétal dans un miel souillé avec pollen en excès (Bahia) (100 x). 

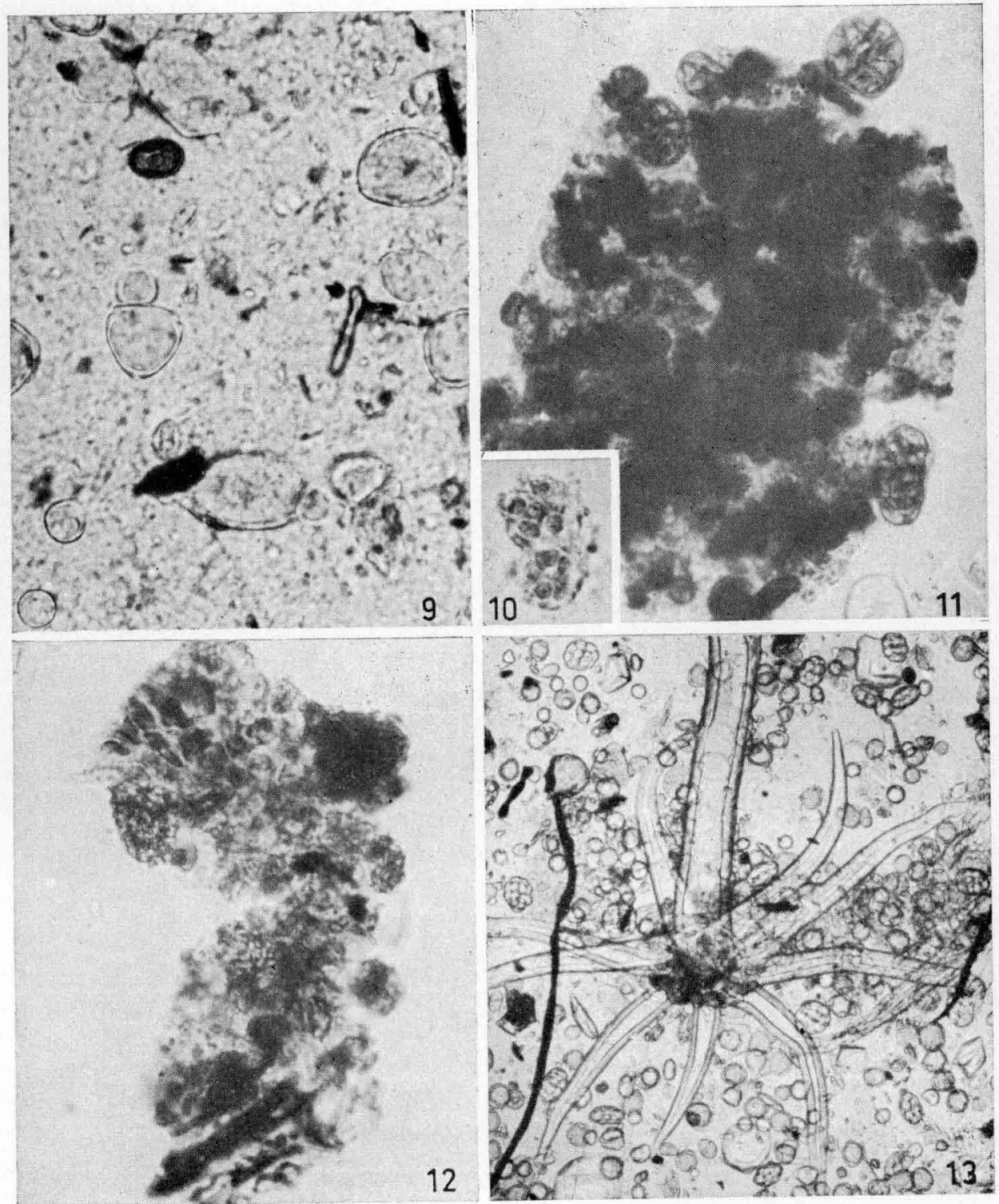

Ortrud Monika BaRTH 


\section{TAFEL III}

Aвв. 14. - Wachs (W), Pflanzenhaar und Brutfragment (B) (Honig aus Paraná) (100 x).

Авв. 15. - Rußschollen und Rußnadeln aus einem verunreinigten Honig mit Pollenüberschuss (Bahia) (100 x).

Aвв. 16. - Rußpartikel aus einem Eucalyptus-Honigtauhonig (Rio de Janeiro).

Авв. 17. - Stärkekörner aus einem stärkereichen und pollenarmen Honig (Rio de Janeiro).

Авв. 18. - Maispollenkörner (Z) und solche des Croton-Typs (C) u. a. m. (Honig aus Rio de Janeiro) (100 x).

Авв. 19. - Cyperaceenpollenkörner (CY) aus einem Honigtauhonig (Bahia). Авв. 20. - Helminthosporium (?) (Honigtauhonig aus Bahia) (1 $000 \mathrm{x}$ ).

Aвв. 21. - Alternaria-Sporen (Honigtauhonig aus Bahia).

Aв8. 22. - Helminthosporium (?) (Honigtauhonig aus Bahia).

\section{PLANCHE III}

Fig. 14. - Cire (W), poil végétal et fragment de couvain (B) (Miel provenant de Paraná) $(100 \mathrm{x})$.

Fig. 15. - Suie en motte et en aiguille dans un miel souillé avec pollen en excès (Bahia) (100 x).

Fig. 16. - Particule de suie dans un miel de miellat d'Eucalyptus (Rio de Janeiro).

FIG. 17. - Grains d'amidon dans un miel riche en amidon et pauvre en pollen (Rio de Janeiro).

Fig. 18. - Pollen de Maïs (Z) et pollen du type Croton (C) (Miel de Rio de Janeiro) $(100 \mathrm{x})$.

Fig. 19. - Grains de pollen de Cypéracées (CY) dans un miel de miellat (Bahia).

Fig. 20. - Helminthosporium (?) (Miel de miellat de Bahia) (1 $000 \mathrm{x}$ ).

Fig. 21. - Alternaria (Miel de miellat de Bahia).

Fig. 22. - Helminthosporium (?) (Miel de miellat de Bahia). 

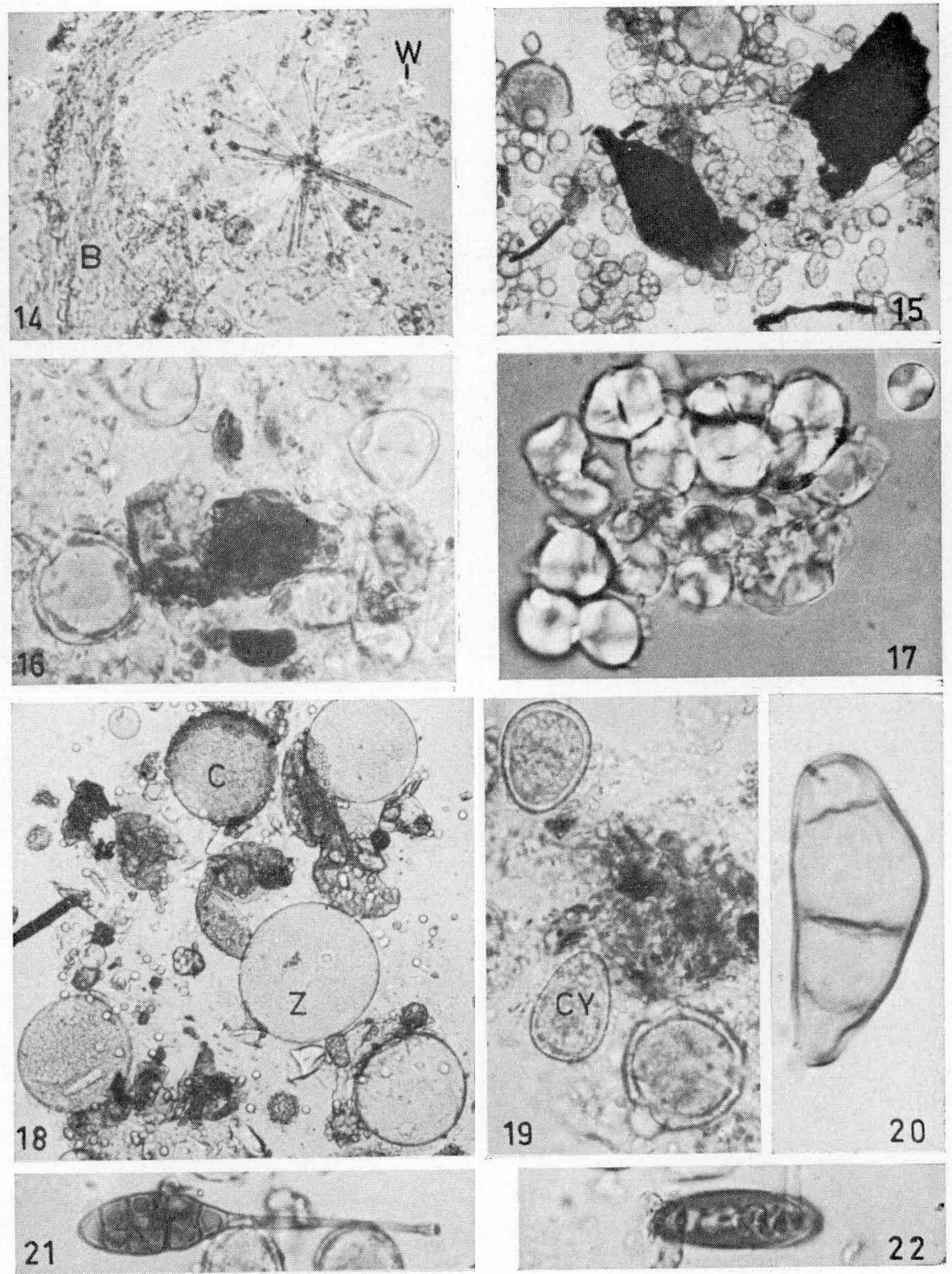